\title{
The Cough Reflex Intensity Score in Critically III Patients' Airway Management: Study Protocol for a Multicenter, Prospective, Observational Trial
}

\section{Wenbin Jia}

Xinjiang Medical University

Jingyi Wang

Peking Union Medical College Hospital

Joseph Harold Walline

The Chinese University of Hong Kong

\section{Ranran Gao}

Xinjiang Medical University

\section{Ran Xu}

Xinjiang Medical University

\section{Xiangya Chen}

Xinjiang Medical University

Xin Yuan

Xinjiang Medical University

Yongkai Li

Xinjiang Medical University

Jian-Zhong YANG ( $\nabla$ yjz6542@126.com )

Xinjiang Medical University

Jun Xu

Peking Union Medical College Hospital

\section{Research Article}

Keywords: airway management, cough reflex, score, endotracheal intubation

Posted Date: June 14th, 2021

DOI: https://doi.org/10.21203/rs.3.rs-167255/v1

License: (1) (1) This work is licensed under a Creative Commons Attribution 4.0 International License.

Read Full License 


\section{Abstract \\ Background}

A patient's ability to cough is important for assessing a patient's airway condition and likely mechanical ventilation outcome,there is still a lack of data comparing patients' initial cough ability and outcomes.

\section{Methods}

The study is a prospective, observational trial which includes 144 patients from Xinjiang Medical University. After a patients is assigned a cough strength score, the cough intensity assessments will be implemented every other day for a week. The primary endpoint is whether the patient requires endotracheal intubation (including tracheostomy). Secondary endpoints include time spent under mechanical ventilation (excluding noninvasive ventilation), ICU and hospital lengths of stay, hospital expenses and in-hospital 30-day mortality.

\section{Discussion}

Anecdotally in our practice, we found that patients with a high Cough Reflex Intensity Score don't require endotracheal intubation, while patients with a low score always need to be intubated. This trial will test to what degree cough intensity is correlated to patient's outcomes.

\section{Trial registration:}

Chinese Clinical Trial Registry,ChiCTR1900028265. Registered 16 December 2019

\section{Background}

Airway management in the care of critically ill patients is of paramount importance. It is a foundational part of treatment for all critically ill patients. Improper airway management can directly threaten patients' lives ${ }^{[1-4]}$, while effective airway management can reduce mortality ${ }^{[24-25]}$. A key element in managing a patient's airway is determining their ability to protect their airway via coughing. A patient's ability to cough is a function of their respiratory center drive and respiratory muscle strength ${ }^{[7]}$, and is important for assessing a patient's airway condition and likely mechanical ventilation outcome ${ }^{[5]}$. At present, a study ${ }^{[8]}$ has classified patients' cough ability according to cough strength and secretions. This classification is divided into three grades: strong, medium, and weak. There have also been four ${ }^{[8,9,12,17]}$ studies which looked for any correlation between a patient's cough ability and the effectiveness of noninvasive mechanical ventilation [10]. These studies demonstrated that the worse the cough ability, the higher their risk of failing noninvasive mechanical ventilation, and the higher their in-hospital mortality ${ }^{[15-16]}$. Some 
studies have tested the efficacy of cough ability to predict extubation outcome ${ }^{[6,8,12-14]}$, which showed that the stronger an ability to cough, the higher the rate of successful extubation ${ }^{[18,19]}$. However, these studies mainly focused on the cough ability of patients receiving mechanical ventilation, there is still a lack of data comparing patients' initial cough ability and outcomes. This will be a prospective, observational study using the Cough Reflex Intensity Score, which is based on a previously proposed cough strength classification $[17,21,23]$. This study will aim to determine whether the Cough Reflex Intensity Score is effective at assessing a patient's risk for airway sputum obstruction, respiratory infection, or mechanical ventilation.

\section{Methods}

\section{Trial design and setting}

The study is a prospective, observational trial which includes 144 patients from Xinjiang Medical University. The Ethics Committee of the First Affiliated Hospital of Xinjiang Medical University approved the trial protocol on August 28, 2019 (reference number K201908-03). And the named ethics committee which approved the study also approved the waiving of/need for consent to participate in the study. The schedule for enrolment, intervention, and assessment will follow the Standard Protocol Items:

Recommendation for Interventional Trials (SPIRIT) guidelines (see Additional File 1) and is presented in Fig. 1.

\section{Population}

Inclusion criteria are: critically ill patients who stay in either the Emergency Resuscitation Unit or the Intensive Care Unit of a hospital. Exclusion criteria are: (1) uncooperative patients, (2) patients with heart failure or multiple organ failure, (3) patients post-cardiopulmonary resuscitation, (4) patients with severe asthma, and (5) pregnant or lactating women.

There is a research coordinator at each hospital to monitor and coordinate the trial. This is a noninterventional study, so informed consent was waived for this observational trial.

\section{Study Tool}

The cough reflex intensity tool, based on the Semi-quantitative Cough Strength

Score first proposed by Khamiees in $2001^{[11]}$, was formulated using the Delphi method ${ }^{\text {[20] }}$

Grade one: No cough. The patient is conscious but has no strength to cough, is unable to make a coughing sound, is unconscious (such as in a coma), is unable to follow instructions, or has no cough reflex when presented with an external stimulation (such as sputum aspiration and pressing on the cricothyroid membrane). 
Grade two: Has a weakly audible cough. The patient is conscious and able to follow instructions, but with only a faint sound and unable to produce sputum out of the glottis.

Grade three: Has a clearly audible cough. The patient can produce a clear coughing sound, able to cough sputum out of the glottis but not out of their mouth.

Grade four: Has a strong, loud cough. The patient can cough sputum out of their mouth.

\section{End-points}

The primary endpoint is whether the patient requires endotracheal intubation (including tracheostomy). Secondary endpoints include time spent under mechanical ventilation (excluding noninvasive ventilation), ICU and hospital lengths of stay, hospital expenses and in-hospital 30-day mortality.

\section{Observation and Date-Collection Procedures}

We will collect patient characteristics (age, gender, weight and height), baseline clinical characteristics (medical diagnosis, past medical and surgical histories, Glasgow Coma Scale, arterial blood gas results and vital signs), as well as cough strength score at patient enrollment.

After a patients is assigned a cough strength score, the cough intensity assessments will be implemented every other day for a week. We will collect vital parameters, including arterial blood gas results, vital signs and Glasgow Coma Scale. Other treatments, such as antibiotics, non-invasive mechanical ventilation, physical nursing treatments (e.g. chest physiotherapy or turning the patient), atomization/nebulization treatments, and any sputum suction results will be recorded.

We will also record the rate of treatment failure, as defined by worsening dyspnea or a respiratory rate $>30$ breaths/min; whether the patients receive endotracheal intubation or tracheotomy; time spent under mechanical ventilation, ICU and hospital length of stay, hospital expenses and in-hospital mortality.

The decision to intubate the patient will be decided by the physician in charge of that patient's care,

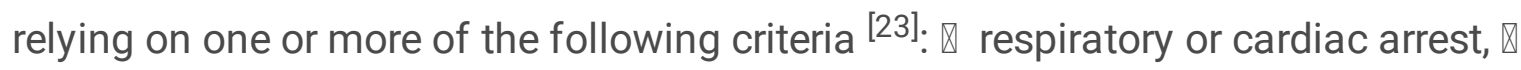
$\mathrm{SpO}_{2} / \mathrm{FiO}_{2}<200 \mathrm{mmHg}$, $\otimes$ apnea with loss of consciousness, $\otimes$ bradycardia (heart rate $<50$ beats $/ \mathrm{min}$ ) with

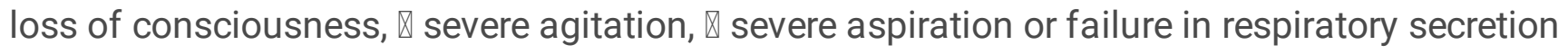

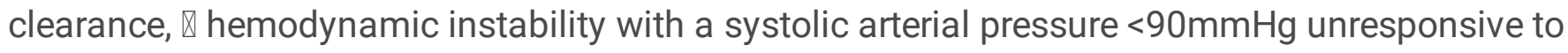

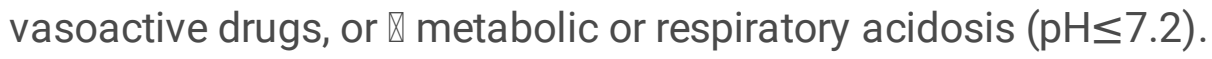

Data will be collected using a standardized case report form. Data will be de-identified before being entered into the database. The study site is regularly monitored, and the database is checked to ensure the accuracy of collected data.

\section{Data analysis}

\section{Sample size calculation}


Sample size was determined based on data from a prospective study ${ }^{[8]}$. The minimal sample size is calculated to be at least 30 participants in each group. In consideration of a possible dropout rate of $10 \%$, we will aim to recruit 144 participants in total.

\section{Data analysis plan}

Analyses will be performed using SPSS version 20.0 or above (SPSS Inc, Chicago, USA). A two-sided pvalue less than 0.05 will be considered statistically significant.

For continuous data, normality tests will first be carried out. If all groups meet the test for normality and the variance between two groups is equal, the homogeneity of the variance will be tested when comparing the groups and the variance analysis will be used to compare the groups. Otherwise, the nonparametric Wilcoxon rank sum test will be applied.

\section{Patients and public involvement}

Patients and the public will not be directly involved in developing research questions or in designing the study.

\section{Discussion}

There is increasing concern about airway management, especially early management in critically ill patients. Potential airway management strategies include: airway assessment, oxygen therapy, artificial airway establishment, maintenance and removal ${ }^{[26]}$. The main purpose of airway management is to maintain good ventilation, ameliorate hypoxia and prevent respiratory failure. A proper respiratory drive, an unobstructed airway, and sufficient respiratory function are essential to achieving spontaneous breathing and preventing hypoxia. Central respiratory dysfunction, airway obstruction, or respiratory insufficiency can cause or exacerbate hypoxia, leading to emergent insertion of an artificial airway.

Advanced airway assessment plays an important role in guiding timely and accurate oxygen therapy or invasive/noninvasive ventilation strategies, which help reduce the reintubation rate of extubated patients

[20]. Serving as a vital part of airway management, cough intensity is closely related to the patient's ability to expel respiratory secretions and prevent airway obstruction.

Anecdotally in our practice, we found that patients with a high Cough Reflex Intensity Score don't require endotracheal intubation, while patients with a low score always need to be intubated. This trial will test to what degree cough intensity is correlated to patient's outcomes.

\section{Trial status}

The protocol version is Version 1.0; 31 January 2020, Recruitment began on January 1, 2020. The expected date for recruitment completion is between May and June 2021. 


\section{Declarations}

\section{Funding $\mathbb{X}$}

The study is funded by Autonomous Region Education Reform Project邓batch number PT2020023区.

Related Articles》

I clarify that none publications containing the results of this study have already been published or submitted to any journal.

Abbreviations $\rrbracket$

N/A

Ethics approval and consent to participate区

The Ethics Committee of the First Affiliated Hospital of Xinjiang Medical University approved the trial protocol on August 28, 2019 (reference number K201908-03). The named ethics committee which approved the study also approved the waiving of/need for consent to participate in the study

Consent for publication $\rrbracket$

The authors agree the publication and claim that none of the material in the paper has been published or is under consideration for publication elsewhere.

Availability of data and material冈

The datasets generated and/or analyzed during the current study are available from the principal investigator (Wenbin Jia) on reasonable request.

Competing interests $\bigotimes$

On behalf of all authors, the corresponding author states that there is no conflict of interest.

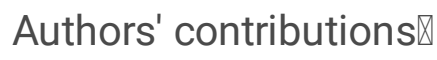

WBJ drafted the manuscript. JYW, RRG and JW co-authored the writing of the manuscript. JZY designed the study and edited the manuscript. JX critically assessed the study design. All authors read and approved the final manuscript.

Acknowledgements $\rrbracket$

N/A 
Trial registration: Chinese Clinical Trial Registry,ChiCTR1900028265. Registered 16 December 2019, http://www.chictr.org.cn/index.aspx.

\section{References}

1. Asai, Takashi. Progress in difficult airway management[J]. Journal of Anesthesia, 2017:1-4.

2. Norris A M , Hardman J G , Asai T . A firm foundation for progress in airway management.[J]. British Journal of Anaesthesia(5):613-616.

3. Lockey D J , Healey B , Crewdson K, et al. Advanced airway management is necessary in prehospital trauma patients[J]. British Journal of Anaesthesia, 2015(4):657.

4. Artime C A , Hagberg C A . Is There a Gold Standard for Management of the Difficult Airway?[J]. Anesthesiology Clinics, 2015, 33(2):233-240.

5. Anderson J, Klock PA Jr. Airway management[J]. Anesthesiology Clinics, 2014, 32(2):445.

6. Coplin W , Pierson D , Cooley K, et al. Implications of Extubation Delay in Brain-Injured Patients Meeting Standard Weaning Criteria[J]. Am J Respir Crit Care Med, 2000, 161(5):1530-1536.

7. Kang S W, Shin J C, Park C I , et al. Relationship between inspiratory muscle strength and cough capacity in cervical spinal cord injured patients[J]. Spinal Cord, 2006, 44(4):242.

8. Gao X J , Qin Y Z . [A study of cough peak expiratory flow in predicting extubation outcome].[J]. Zhongguo W Zhong Bing Ji Jiu Yi Xue, 2009, 21(7):390-393.

9. Duan J , Zhou L , Xiao M , et al. Semiquantitative Cough Strength Score for Predicting Reintubation After Planned Extubation[J]. American Journal of Critical Care, 2015, 24(6):e86-e90.

10. Meiling $X$, Jun $D$, Respiratory $D O$. Association between cough strength and the efficacy of noninvasive mechanical ventilation in patients with respiratory failure[J]. China Medical Herald, 2018.

11. Mohammad Khamiees, Priya Raju, Angela DeGirolamo, Yaw Amoateng-Adjepong, and Constantine A. Manthous. Predictors of extubation outcome in patients who have successfully completed a spontaneous breathing trial[J]. Chest, 2001, 120(4):1262-1270.

12. Smina M , Salam A, Khamiees M, et al. Cough peak flows and extubation outcomes.[J]. Chest, 2003, 124(1):262-268.

13. Thille A W , Boissier F, Ben Ghezala H, et al. Risk factors for and prediction by caregivers of extubation failure in ICU patients: a prospective study.[J]. Critical Care Medicine, 2015, 43(3):613-20.

14. Guru P K, Singh T D , Pedavally S, et al. Predictors of Extubation Success in Patients with Posterior Fossa Strokes[J]. Neurocritical Care, 2016, 25(1):117-127.

15. Xu X P , Zhang X C , Hu S L , et al. Noninvasive Ventilation in Acute Hypoxemic Nonhypercapnic Respiratory Failure[J]. Critical Care Medicine, 2017, 45(7):e727.

16. Chandra D , Stamm J A, Taylor B, et al. Outcomes of noninvasive ventilation for acute exacerbations of chronic obstructive pulmonary disease in the United States, 1998-2008.[J]. American Journal of Respiratory \& Critical Care Medicine, 2012, 185(2):152. 
17. Ibrahim A S , Aly M G , Abdel-Rahman K A, et al. Semi-quantitative Cough Strength Score as a Predictor for Extubation Outcome in Traumatic Brain Injury: A Prospective Observational Study[J]. Neurocritical Care, 2018.

18. Jiao-Hua W U , Jin-Qing $L$, Hua-Qiong $H$, et al. Effectiveness evaluation of active cycle of breathing techniques on the autonomously expectoration ability in patients after tube drawing[J]. Internal Medicine of China, 2014.

19. Min D , Linfu B , Jun D . Efficacy of preventive use of noninvasive ventilation in planned extubated patients with weak cough[J]. Journal of Chongqing Medical University, 2017.

20. Machado K F , Maciel D A, Mello R M D , et al. Reflex cough PEF as a predictor of successful extubation in neurological patients[J]. Jornal Brasilro De Pneumologia, 2015, 41(4):358-364.

21. Fan $L$, Zhao Q , Liu $Y$, et al. Semiquantitative cough strength score and associated outcomes in noninvasive positive pressure ventilation patients with acute exacerbation of chronic obstructive pulmonary disease[J]. Respir Med, 2014, 108( 12):1801-1807.

22. Mohammad Khamiees, Priya Raju,Angela DeGirolamo, Yaw Amoateng-Adjepong, and Constantine A. Manthous. Predictors of extubation outcome in patients who have successfully completed a spontaneous breathing trial[J]. Chest, 2001, 120(4):1262-1270.

23. Zhi-Qiang Q . Indications for mechanical ventilation in patients with common respiratory diseases[J]. New Medicine, 2011.

24. Eckstein M , Chan L, Schneir A, et al. Effect of prehospital advanced life support on outcomes of major trauma patients.[J]. Journal of Trauma and Acute Care Surgery, 2000, 48(4):643.

25. Meizoso J P, Valle E J , Allen C J , et al. Decreased mortality after prehospital interventions in severely injured trauma patients[J]. Journal of Trauma and Acute Care Surgery, 2015, 79(2):227-231.

26. Overbeck, Michael C . Airway Management of Respiratory Failure.[J]. Emergency Medicine Clinics of North America, 2016, 34(1):97.

\section{Supplementary Files}

Additional File 1 is not available with this version

\section{Figures}




\begin{tabular}{|c|c|c|c|c|c|c|c|}
\hline \multirow[b]{3}{*}{ TIMEPOINT T* } & \multicolumn{7}{|c|}{ STUDY PERIOD } \\
\hline & \multirow{2}{*}{$\begin{array}{c}\text { Enrolment } \\
-t_{1}\end{array}$} & \multirow{2}{*}{$\frac{\text { Allocation }}{0}$} & \multicolumn{4}{|c|}{ Post-allocation } & \multirow{2}{*}{$\begin{array}{c}\text { Close-out } \\
\text { Hospital } \\
\text { Discharge }\end{array}$} \\
\hline & & & $\begin{array}{c}\text { Day } \\
1\end{array}$ & $\begin{array}{c}\text { Day } \\
3\end{array}$ & $\begin{array}{c}\text { Day } \\
5\end{array}$ & $\begin{array}{c}\text { Day } \\
7\end{array}$ & \\
\hline \multicolumn{8}{|l|}{ ENROLMENT: } \\
\hline Eligibility screen & $X$ & & & & & & \\
\hline Informed consent & $X$ & & & & & & \\
\hline Allocation & & $X$ & & & & & \\
\hline \multicolumn{8}{|l|}{$\begin{array}{r}\mathrm{NO} \\
\text { INTERVENTIONS: }\end{array}$} \\
\hline \multicolumn{8}{|l|}{ ASSESSMENTS: } \\
\hline \multirow{2}{*}{$\begin{array}{r}\text { characteristics } \\
\text { baseline clinical } \\
\text { characteristics }\end{array}$} & $X$ & & & & & & \\
\hline & $X$ & & $X$ & $X$ & $X$ & $X$ & $X$ \\
\hline \multirow{3}{*}{$\begin{array}{r}\text { Primary outcome } \\
\text { Secondary } \\
\text { outcome }\end{array}$} & & & $\mathrm{X}$ & $x$ & $x$ & $\mathrm{X}$ & $\mathrm{X}$ \\
\hline & & & & & & & $X$ \\
\hline & & & $\mathrm{X}$ & $\mathrm{X}$ & $X$ & $x$ & $x$ \\
\hline
\end{tabular}

Figure 1

Standard Protocol Items: Recommendation for Interventional Trials (SPIRIT) schedule of enrolment, intervention and assessments. Characteristics: age, gender, weight and height. Baseline clinical characteristics :(medical diagnosis, past medical and surgical histories, Glasgow Coma Scale, arterial blood gas results and vital signs. Primary outcome:endotracheal intubation. Secondary outcome:time spent under mechanical ventilation (excluding noninvasive ventilation), ICU and hospital lengths of stay, hospital expenses.Other treatments:antibiotics, non-invasive mechanical ventilation, physical nursing treatments (e.g. chest physiotherapy or turning the patient), atomization/nebulization treatments, and sputum suction. 


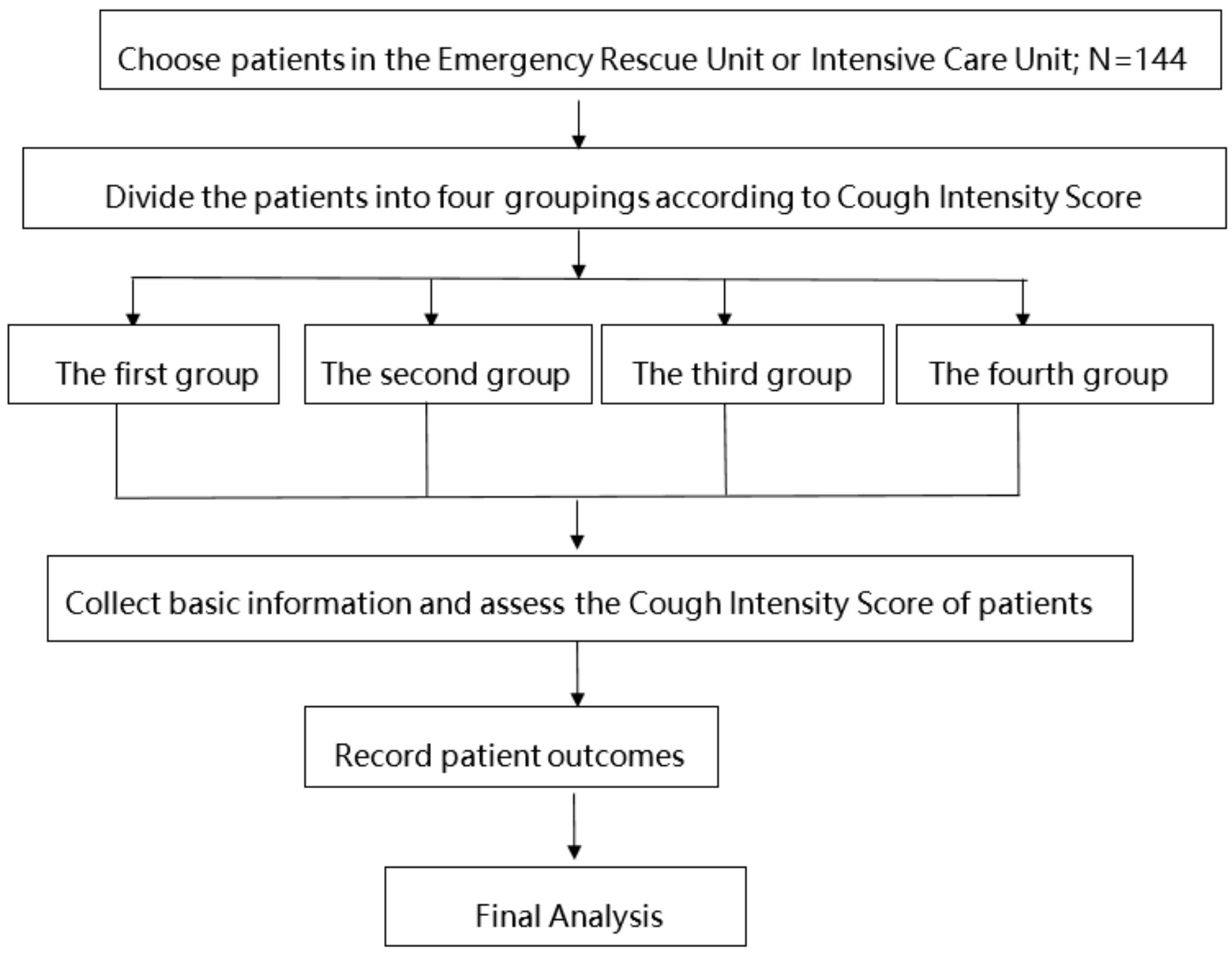

Figure 2

Technology Roadmap 